\title{
AlloHaptic: Robot-Mediated Haptic Collaboration for Learning Linear Functions*
}

\author{
Hala Khodr ${ }^{1}$, Soheil Kianzad ${ }^{2}$, Wafa Johal ${ }^{1,3}$, Aditi Kothiyal ${ }^{1}$, Barbara Bruno ${ }^{1,4}$ and Pierre Dillenbourg ${ }^{1}$
}

\begin{abstract}
Collaborative learning appears in a joint intellectual efforts of individuals to understand an object of knowledge collectively. In their search for understanding the problems, meanings, and solutions, learners employ different multi-modal strategies. In this work, we explore the role of force feedback in learners interaction with tangible hand-held robots. We designed a collaborative learning environment to provide embodied intuitions on linear mathematical functions combined with graphical representations and ran a first study involving 24 participants. Our analysis shows a positive learning gain for our learning activity. Moreover, to explore the link between different types of force feedback and learners' collaboration, we designed a focus group study with 12 participants. Our results suggest that the haptic communication channel affects the collaboration dynamic differently according to the nature of the learning task. We finish by proposing design insights for future exploration of haptic in collaborative learning.
\end{abstract}

\section{INTRODUCTION}

Ever since the introduction of robots in educational contexts, one of the most fundamental and yet elusive questions tackled by HRI researchers has been: Is the robot ultimately helping learners collaborate with each other, or not?

The question is important for two reasons. From a practical perspective, collaborative learning activities, i.e., situations in which two or more people learn or attempt to learn something together [1] are the usual setting of robotic activities in classrooms (also because of the common disparity between number of children and number of robots): to know whether and how robots are a good fit for their context is a necessary prerequisite for the effective introduction of robots in learning activities. From a theoretical perspective, literature in education provides ample examples of the intuition that collaboration among learners can have a positive effect on motivation and social skills as well as on learning [2]. As a consequence, the design of robots to maximize the probability that the collaboration among learners generates learning should be a priority in educational robotics.

Two approaches can be found in the literature concerning the way the robot should facilitate collaboration among learners. Explicit solutions typically envision a social robot to act as an instructor or peer for the learners, and rely on its behaviour to promote collaboration [3], [4], [5]. Conversely,

*This work has been supported by the Swiss National Science Foundation through the National Centre of Competence in Research Robotics.

1 Authors are with the Computer Human Interaction in Learning and Instruction Laboratory (CHILI), Swiss Federal Institute of Technology (EPFL), Lausanne, Switzerland, hala.khodreepfl.ch

S. Kianzad is with the 2 Department of Computer Science at the University of British Columbia

W. Johal is with the ${ }^{3}$ University of New South Wales, Sydney, Australia

B. Bruno is also with ${ }^{4}$ MOBOTS Group in BIOROB, EPFL, Lausanne

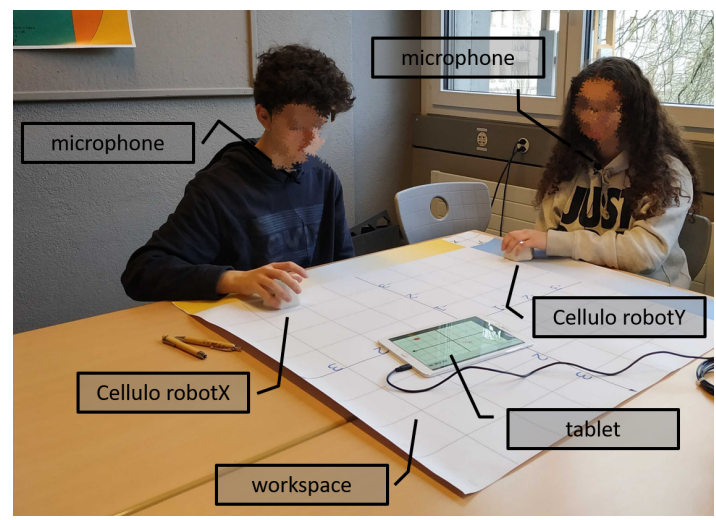

Fig. 1: Activity setup

implicit solutions typically envision the robots to be tools in the hands of children, and frame the learning activity to rely on the robots' intrinsic characteristics and functionalities to promote collaboration [6].

Within the latter category, Cellulo (held by children in Fig. 1) is a small, tabletop robot specifically developed to support learning [7], which includes, among its functionalities, the possibility to provide haptic feedback to the person holding it. We see this as an opportunity to create a non-verbal communication channel among learners to haptically speak to each other through their robots.

In this article, we investigate the role of haptics in collaborative learning activities involving robots. Specifically, the contribution of this article is two-fold. In the first study, we propose and evaluate a learning activity which exploits robots and haptics to help learners collaborate and obtain an intuitive understanding of mathematical concepts. The results of this study suggest that the relation between haptics, robots and learning is not straightforward. In the second study, we dig deeper into that relationship, specifically investigating the role of haptics towards collaboration and coordination among learners.

\section{RELATED WORK}

Computer Supported Collaborative Learning (CSCL) investigates how technology can support or mediate collaborative knowledge construction and problem solving [8]. The field has grown in the past 15 years demonstrating its applicability in classroom settings [9]. One important aspect of CSCL is to design a digital medium that enables communication between learners without time and distance constraints. Communication is essential in order to build a shared understanding and a common ground. When 
synchronous, communication mediums also allow to bring awareness to collaborators.

Within the interests of CSCL in exploring remote collaboration, researchers have proposed novel designs to enhance learners' communication, such as through writing text, visual cursor and video-conferencing [10]. However, the use of the haptic communication channel among learners still remains unexplored. Haptic collaboration appears in a wide range of activities from furniture handling [11] to a more advanced form of teleoperation [12]. This allows users to manipulate objects [11], share experiences [13], and communicate their emotions [14].

Through haptics, users can seamlessly communicate essential collaborative information [15], raising awareness [16] and aiding users to coordinate their actions [17]. The intrusive information from the haptic channel connects users to the environment and to their collaborator, thus potentially enhancing their mutual understanding of a task or a shared goal [11], [9].

In addition to the communicative benefits, a large body of work explores the advantages of adding haptic feedback in non-collaborative learning activities. In most of the learning studies with haptics, the task is performed alone by learners [18], [19], [20]. Some researchers ground their studies on the theories of embodied cognition and investigate the impact of haptics on enhancing students' learning experience [21], [22], [23]. On the other hand, a group of researchers considered haptics as a key sensory input channel to receive crucial information from the learning environment [24], [25].

We can also separate the common types of educational activities with haptics into three categories: Motor control skills- to acquire or to improve handwriting [20], [26], haptic mirror- to mimic physical experience [23], [27], and haptic bridge- to connect a dynamic visualization of a mathematical concept with a haptic representation [28], [29], [30], [19].

Despite encouraging findings, there is still a large inconsistency in the results of haptic studies wherein some researchers could not find learning advantages of adding touch sensory input [31], [32], [25]. Zacharia [33] suggests the need for establishing a framework on when, why, and how haptic should be employed in learning activities. More studies are required to establish the mentioned framework and to unpack the details of students' conceptualization through haptics. In this study, we will look at how haptic as a communication channel can impact the collaborative learning efforts among peers.

Mathematics is special field of science that contains the most abstract concepts among other fields. Several studies explore the possibility of improving children conceptualization of mathematical concepts by making the abstract more tangible [34], [35]. Bodily gestures are considered as a rich source of information that both students and teachers make mostly unconsciously while expressing graphs [36], [34]. These studies indicate that students with robust mathematical conceptualization use verbal/kinesthetic/visual cues to express their imagination of graphs [37]. For these reasons, we chose Mathematics as the subject to design a collaborative and haptic learning activity. While most of the haptic devices suffer from a small workspace as they need be anchored to the ground, a large workspace haptic device such as the one we describe in this work, can potentially enable a full body interaction with the mathematical concepts and further enhance learning.

\section{Material AND Methods}

The Cellulo platform consists of three main components:

1) The workspace, consisting of printed sheets of paper "augmented" with a dot pattern [7]. The paper size, as well as its graphics, are defined in accordance with the activity requirements.

2) The Cellulo robots, handheld haptic-enabled mobile robots, capable of holonomic motion and absolute global localization (position and orientation $(x, y$, theta) $)$ within the workspace. When placed on the "augmented" paper sheet, each robot can self-localize with sub-mm accuracy via an image sensor placed underneath the robot. Moreover, each robot has a top surface equipped with 6 capacitive touch sensors as well as 6 full RGB LEDs which allow for simple visual and touch interaction. Finally, the locomotion drive system was designed to be robust against intensive physical user interaction, allowing the robot to be used to render haptic feedback and to exert a force of about 1N [38]. Details on the electronic and mechanical design are found in [7].

3) The central controller (either a desktop computer or a consumer-grade tablet), running a cross-platform QtQuick application which contains the logic of the activity and coordinates the movements of the robots through a star network composed of point-to-point Bluetook SPP links. Each robot connects wirelessly to the central controller, reports to it all events (e.g pose changed) and receives from it commands (e.g. track a given velocity).

The setup of the activity, shown in Fig. 1, involves a team of two children: each one has a robot (denoted as robot $X$ and $\operatorname{robot} Y$ ), and collaboratively they control a third, virtual robot, the team robot, on the tablet that also serves as central controller. RobotX is solely responsible for the team robot $\mathrm{x}$ coordinate, while robot $\mathrm{Y}$ is solely responsible for the team robot y coordinate. During the activity, the logs of robotX and robotY (position and orientation, sensor status) are recorded and lavalier microphones are attached to the participants' clothes to record their utterances, in order to analyze the verbal exchange within the team.

We distinguish between two types of force feedback that can be provided to each participant:

1) Corrective Haptic Feedback: It is computed as a force in the direction allowing the team robot to get to the target curve (Fig. 2). Specifically, the force is calculated in the direction of the closest point of the curve as: $\vec{F}=k_{c}\left(\vec{r}_{c}-\vec{r}_{v}\right)$ where $k_{c}$ is a constant for tuning the coupling strength, $\vec{r}_{c}$ is the point along the target curve closest to the team robot current position, and $\vec{r}_{v}$ is the current position of the team robot, equal to $\vec{r}_{v}=\left(x_{\text {robot } X}, y_{\text {robot } Y}\right)$.

2) Assistive Haptic Feedback: It is computed as a force allowing the team robot to move along the target curve when 


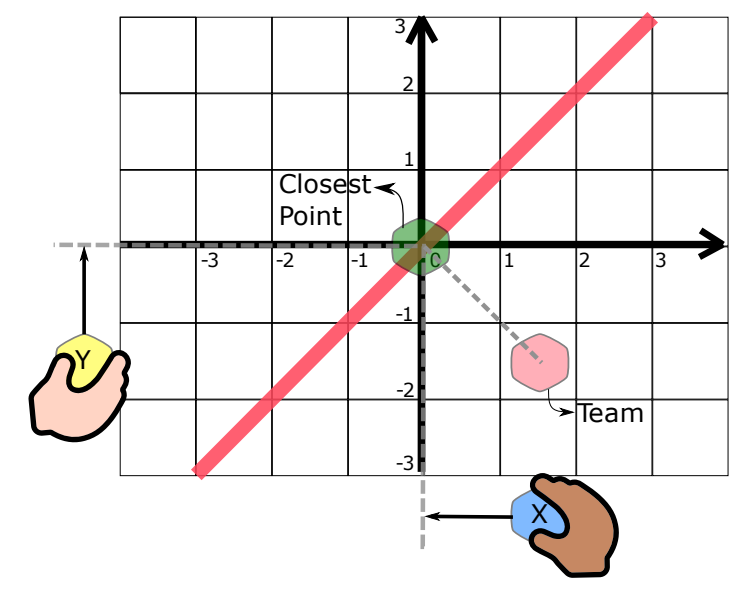

Fig. 2: Corrective haptic feedback "pushes" participants' robots to keep the team robot (pink) on the target line. In this case, robotX (blue) is pushed to the left while robotY (yellow) is pushed upwards. In this example, the function $y=x$ is illustrated by the red line which was or not actually printed on the water-marked paper and would change according to the task.

on it. Specifically, the force is calculated as: $\vec{F}=k_{a} v_{d} \vec{T}_{\vec{r}_{v}}$ where $k_{a}$ is a constant tuning the force strength, $\vec{T}$ computes the normalized tangent direction of the point on the curve (i.e, the gradient vector in the case of a line) and $v_{d}$ is the desired speed to traverse the curve, set as $50 \%$ of the maximum speed of a Cellulo robot. When outside the curve, a corrective feedback can be further added.

In both cases, $\mathrm{F}$ is then decoupled between the two participants so that the force applied to $\operatorname{robotX}$ is $\left(F_{x}, 0\right)$ and the force applied to robotY is $\left(0, F_{y}\right)$.

We employ the described setup in our learning activity, aiming to lead participants to acquire an intuitive understanding of the notion of function in a collaborative way. We run two studies. The first study focuses on evaluating the learning goal of the activity, additionally investigating whether the presence of the robot (i.e., a tangible element in the intangible context of mathematical functions) is beneficial, detrimental, or negligible towards learning. Building upon the findings of study 1 , the second study investigates the potential of haptic feedback as a communication channel between two users engaged in the collaborative learning activity.

\section{STUDY 1}

As stated above, the main focus of the first study was to assess the efficacy of the proposed activity with respect to its learning objectives. Concretely, this translates into the following Research Questions:

RQ1 Is the interaction with the robots beneficial for learning? Specifically, [RQ1.1] is there a positive correlation between the presence of haptic feedback and learning? [RQ1.2] is there a positive correlation between the presence of haptic feedback and robot usage? [RQ1.3] is there a positive correlation between robot usage and learning?
RQ2 Does the proposed activity lead to a positive learning gain on concepts related to line functions (specifically, slope and intercept)?

We hypothesise the answer to all above questions to be "yes".

\section{A. Learning Activity}

The activity is composed of two parts: in the first one, participants familiarize with the robots and their respective contributions to the movements of the team robot, by making the team robot move around a circle. In the second part, they use the robots to explore the notions of the slope and the intercept of a line. Iteratively, a line appears on the tablet and participants are asked to answer the following consecutive questions:

1) When robot $X$ is moving by 1 unit in the positive direction, robotY should move by $[\ldots]$.

2) When robot $X$ is at 0 , $\operatorname{robot} Y$ should be at $[\ldots]$

3) We can then write the relation between the two robots as: $\operatorname{robot} Y=[\ldots] \operatorname{robot} X+[\ldots]$

The second part was composed of a sequence of 4 lines, with different slopes and intercepts.

\section{B. Experimental Procedure}

We designed Study 1 as a between-groups design, with a control condition with no feedback (denoted as Passive) and an experimental condition (denoted as Haptic), in which participants feel the corrective haptic feedback explained in Section III.

The study involved 24 participants randomly teamed in pairs. They were 12 to 15 years old girls (mean age $=13.42$, std=0.72), visiting EPFL as a part of a summer camp. The teams were split equally and randomly to the two conditions. Individual pre-test and post-test were conducted to measure the learning gain. Specifically, both the pre-test and posttest included 4 questions, asking to match a given equation to the correct line among the displayed ones, or asking to write the equation of the displayed line. Pre-test and post-test questions differed only in the line parameters' values.

\section{Results}

We calculate the learning gain as the percentage difference between pre- and post-test scores. Fig. 3a shows the distribution of the pre- and post- test scores of the participants with respect to the feedback condition. 15 participants had no prior knowledge (scoring 0 on the pre-test) on functions. As the Figure shows, despite one participant (9y) showing a negative learning gain, 9 participants without prior knowledge learned, and 3 participants with prior knowledge improved their scores.

We noticed a statistically significant increase in the scores when comparing post- and pre-test in both the groups (Fig. 3b). However, the learning gain was not significantly different between the two feedback conditions, nor was the interaction, i.e, the difference in learning gain for the types of feedback (Table I). 


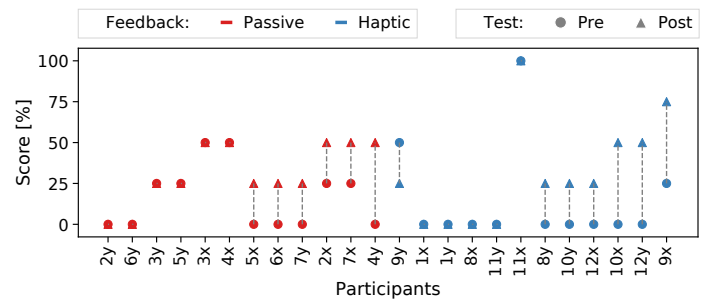

(a)

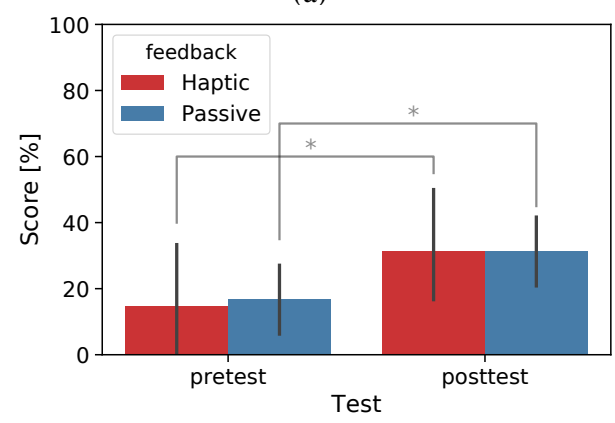

(b)

Fig. 3: Learning Gain Results of Study 1. * indicates a significant difference with $\mathrm{p}$-value $<0.05$ with t-test. The average learning gain in the Haptic condition is $16.6 \pm 24.6$ $\%$ and in the Passive condition is $14.6 \pm 16.7 \%$

TABLE I: Repeated measures ANOVA results. Effect size indicates the partial eta-square effect size.

\begin{tabular}{c|c|c|c} 
Source & F-value & p-value & Effect size \\
\hline Test & 13.2 & 0.0014 & 0.38 \\
Feedback & 0.011 & 0.91 & 0.001 \\
Interaction & 0.06 & 0.81 & 0.003
\end{tabular}

In the context of RQ1, to investigate the interaction with the robots, we define a "robot usage" metric which is equal to the average cumulative distance of the movements of each robot before participants start to answer the inside-activity questions. We study this metric from two aspects: its effect on the learning gain on one hand, and its correlation to the feedback condition, on the other hand. We hypothesize that the presence of haptics will motivate the use of the robot (hence, participants in the Haptic condition would use the robot more than participants in the Passive condition). Results of a t-test show that this distance is significantly higher in the Haptic condition (605.5 $\pm 446.0 \mathrm{~mm}$ vs $198.5 \pm 133.7 \mathrm{~mm}$ with a p-value=0.006), which is in line with the hypothesis that haptics encourages the use of the robots. From a learning gain perspective, we found a positive correlation between students who moved the robot more and their learning gain, although this correlation is only statistically significant in a confidence interval of $95 \%$ (pearson correlation $=0.34, \mathrm{p}$-value $=0.1$ ). We conduct a

TABLE II: Statistical mediation analysis

\begin{tabular}{c|c|c|c|c|c|c} 
Path & Coef & SE & p-value & CI[5.5\%] & CI[94.5\%] & Sig. \\
\hline $\mathrm{F} \rightarrow \mathrm{D}$ & 406.95 & 134.40 & 0.01 & 183.11 & 630.79 & Yes \\
$\mathrm{D} \rightarrow \mathrm{L}$ & 0.02 & 0.01 & 0.10 & 0.00 & 0.04 & Yes \\
Total & 2.08 & 8.59 & 0.81 & -12.22 & 16.39 & No \\
Direct & -7.53 & 9.72 & 0.45 & -23.76 & 8.69 & No \\
Indirect & 9.62 & 5.65 & 0.09 & 0.63 & 17.80 & Yes
\end{tabular}

statistical mediation analysis between the feedback condition and the learning gain where the robot usage metric acts as a mediator variable since it is affected by the feedback condition, and it in turn affects the learning gain. The results are shown in table II. Although we have no evidence of the total effect of the feedback condition $(F)$ on the learning gain (L), this analysis shows a positive indirect effect through the robot usage (D), and that is a conjunction of the effect of $F$ on $\mathrm{D}$ and the effect of $\mathrm{D}$ on $\mathrm{L}$ (with a confidence of $95 \%$ ).

\section{Discussion}

The results of Study 1 preliminarily support RQ2, suggesting that indeed the proposed activity can be effective in providing children with an intuitive understanding of the notions of slope and intercept of a line.

Concerning RQ1, although no significant difference was found between the two conditions in terms of learning gain (RQ1.1), thus suggesting that the haptic feedback is not directly correlated with learning, statistical mediation analysis shows a marginally significant (with a confidence of 95\%) indirect effect though the robot usage metric. Specifically, results on robot usage support RQ1.2, suggesting that haptic feedback indeed motivates participants to interact with the robots. Results marginally support RQ1.3, i.e. a correlation between learning and robot usage exists but only with a more relaxed confidence interval which can be explained by the small sample size and hence small power. Combining these results, we conclude that haptic feedback leads participants to use the robot more, and this, in turn, seems to be correlated with learning, although our results are only showing trends towards significance and therefore merit further investigation.

\section{STudy2}

The results of Study 1, specifically the findings of RQ1, motivate us to further investigate how robot interaction with haptic feedback leads to learning. Research in CSCL [39] suggests the productivity of interactions during collaboration that ultimately leads to learning. This motivates us to investigate further the role haptic feedback plays in communication and collaboration, and to identify the types of interactions during the activity. The experimental setup and feedback types presented in Section III remain the same. Concretely, we are interested in the following Research Questions (RQs):

RQ1 How do participants collaborate and coordinate within the activity?

RQ2 Is there a relation between haptic feedback, collaboration and coordination?

\section{A. Learning scenario}

The goal of this learning activity is for participants to develop an understanding of the sign and strength of a function's slope.

The learning activity is composed of three types of tasks. The first task is named "Find the line": an invisible line is embedded in the workspace, and the team robot will leave a trace on the tablet only if it is on the line. Corrective haptic feedback is given to the participants, and the starting point 
is always at the origin of the coordinate system shown on the tablet and workspace map. The second task is named "Feel the slope". In this task, the line is shown on the tablet, and the goal is to track it. Assistive haptic feedback is given to the participants, thus giving them the impression of feeling the gradient. Finally, the third task is named "Draw the line". Two points are shown on the tablet and the goal is to connect them with a straight line drawn by the team robot. The participants were asked to first predict how they should move, act and then reflect. Corrective haptic feedback is given in this case. This sequence of activities is repeated for 3 examples of lines with positive, negative and steeper slopes.

\section{B. Experimental Procedure}

Study 2 is a within-subjects design, in which all participants go through all the tasks and alternatively experience the corrective and assistive haptic feedback detailed in Section III.

The study involved 12 participants randomly teamed in pairs. They were 13 to 15 years old students (mean age $=14$, $\mathrm{std}=0.66$ ). The experiment took place in a school, during a math session, and lasted approx. 40 minutes.

\section{Quantitative Results}

To investigate the RQs, we define the following metrics as operationalizations of coordination and collaboration.

1) Co-Movement: We introduce the co-movement metric to study the coordination between the two participants, defined as the percentage of time the two participants are moving their robots in the "good" direction to be on the line (i.e., intuitively, how coordinated they are in seeking to reach the task goal). To compute this metric, we first normalize the velocities of robotX and robotY by the slope of the corresponding line. The co-movement is then determined by $\operatorname{sign}\left(v_{x}\right) \odot \operatorname{sign}\left(v_{y}\right)$ where $\odot$ represents a logical $\mathrm{XNOR}$, and $v_{x}, v_{y}$ are the velocities of robotX and robotY respectively.

Fig. 4a shows the average percentage of co-movement with respect to the task type. We observe that the co-movement is significantly less in the find condition when compared to the two others. This could be explained by the fact that only in the find task, there is no visual persistence of the shared knowledge. Previous research in collaborative learning have found that learners used the persistence of grounded information to better collaborate [40].

2) Turn-Taking (Leader-Follower Roles Exchange): The second metric we compute is the emergence of the leaderfollower behavior and specifically the exchange of the roles of leader and follower between participants. To operationalize the leader-follower behavior we track the changes in directions of the robots and tag as the leader the robot that initiated a change that was then followed by the other robot. We then count how many times a pattern of leader switching between robotX and robotY occurs and we denote this as the turn-taking metric. Intuitively, this metric reflects how balanced is the collaboration between the participants.

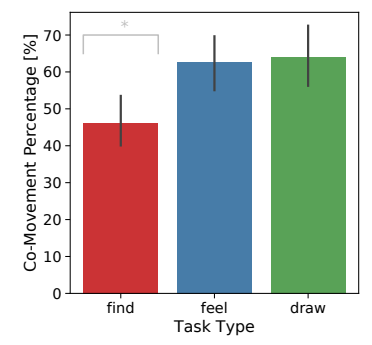

(a)

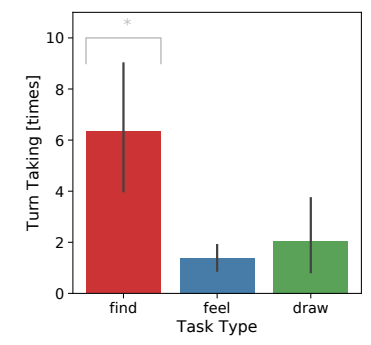

(b)
Fig. 4: (a) Co-movement analysis. (b) Turn-taking analysis. * indicates a significant difference with $\mathrm{p}$-value $<0.05$.

Fig. $4 \mathrm{~b}$ shows the average number of role reversals with respect to the task type.

As the Figures show, participants are encouraged to switch leader and follower roles in the "find" task, whereas the "feel" and "draw" tasks encourage coordination.

3) Individual Response to Haptic Feedback: In order to investigate how a participant responds to the haptic feedback given, we introduce a status metric denoted "agreement with haptic", that can take one of three possible values: \{agree; resist; disagree $\}$. A participant is in the agree state if his/her robot's actual velocity agrees with the direction and intensity of the haptic feedback given (i.e., if he/she is following the haptic guidance). Conversely, a participant is in the resist state whenever the robot's velocity goes in the same direction suggested by the haptic feedback, but not its intensity. Lastly, a participant is in the disagree state whenever he/she is voluntarily moving the robot in the opposite direction of the given feedback.

Fig. 5 shows the mean percentage of agree, resist and disagree time for each individual. We obtain an average of $68 \%$ agreement time, $7 \%$ for resist time and $25 \%$ of disagree time.

We further analysed the effect of the task type on the participant's agreement with haptic. Fig. 6a shows that there was a significantly higher participant's agreement with the haptic feedback in the case of the "feel" activity. We hypothesise that this might be related to the different type of feedback provided in the tasks, with the assistive haptic feedback effectively acting as a "trustworthy guide" for participants.

Finally, we study the correlation between the average (between $\mathrm{x}$ and $\mathrm{y}$ ) haptic agreement metric and the team co-movement metric. We notice a significant positive correlation: Spearman correlation $(0.48)$ and $(p<0.005)$. Fig. $6 b$ shows the correlation with the regression line with $\mathrm{r}=0.23$.

4) Manipulation measures: Accuracy and Velocity: The velocity-accuracy trade-off is a well known metric in object manipulation and tend to be inversely correlated. As one moves fast, the motion is less precise. In order to assess the usability and manipulation of the robot's during the task, we compute the speed of the virtual robot by combining the two axial velocities of robotX and robotY. The accuracy error is given by the distance of the virtual robot to the line. Looking at the correlation between error in accuracy and velocity of the virtual robot, we see that they are significantly 


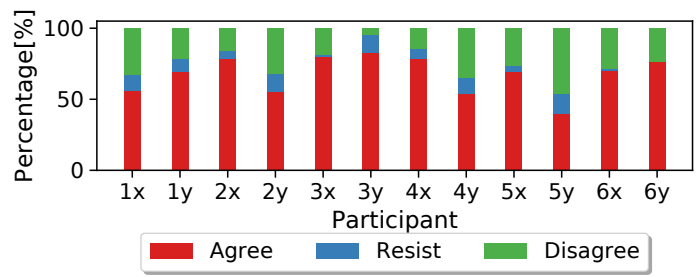

Fig. 5: Individual response to haptic feedback. Participant IDs include the team number and an $\mathrm{x}$ or $\mathrm{y}$ according to the robot they were using.

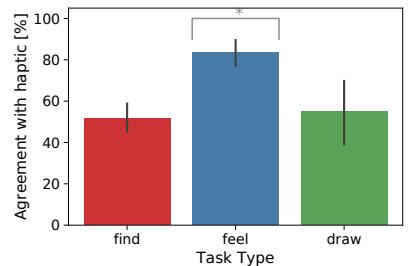

(a)

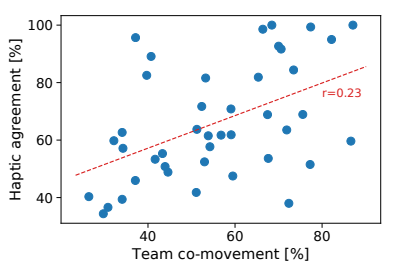

(b)
Fig. 6: Haptic Feedback Analysis. * indicates a significant difference with $\mathrm{p}$-value $<0.05$.

positively correlated (Spearman correlation $=0.38$ and $\mathrm{p}<$ 0.005). Fig. 7 shows also that speed and accuracy error seem to decrease from the find task to the feel task and from the feel task to the draw task. A paired t-test reveals that the speed significantly decreases throughout the tasks, teams going slower towards the draw task. The data for the accuracy error does not give significant difference between the tasks.

\section{Qualitative Results}

We used Braun and Clark's recommended approach to thematic analysis to identify meaningful patterns in dyads' conversations during the activity [41]. Two of the lead investigators conducted an open coding on the transcribed conversations while analyzing the screen recordings. Another member of the research team was collaboratively checking the memos and connecting the codes to describe the interaction with robots in an iterative process. We converge to the themes around the implication of haptics in collaboration.

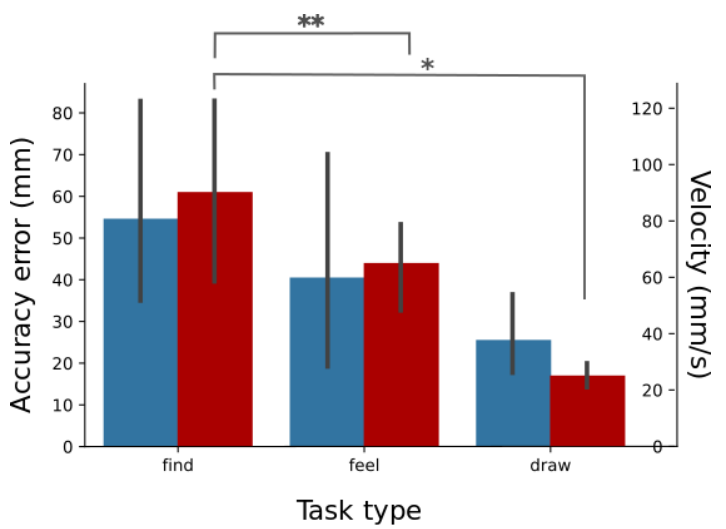

Fig. 7: Average team velocity (in blue) and accuracy error (red) for each task. * and ** indicates a significant difference with $\mathrm{p}$-value $<0.05$ and 0.01 respectively.
1) General observations: Learners began by using the robots as tools for play and exploration during the introductory activities. They felt more confident when they backed their hypotheses by running evaluations with the robots during the learning activities. Thus they gradually achieved co-ordination and understanding of the concepts. We elaborate their strategies below.

2) Agreement with haptic feedback: All learners took some time to understand the nature of the haptic feedback. Eventually however, the corrective haptic feedback helps learners to understand the exact location of the robots on the graph, e.g., when dyads try to find the amount of y when $\mathrm{x}$ is at zero (intercept). The understanding of slope and the relationship between the speeds of robotX and robotY started in the feel task and is completed in the draw task.

3) Collaboration strategies: Learners used vocal channels to initiate their collaboration. They usually counted from three to one to start the task simultaneously. In all the three tasks, we observed that learners moved slowly to coordinate more accurately as also observed from the quantitative accuracy-velocity findings.

$$
\begin{aligned}
& \text { T3-P1: ...start at the right coordinates and go } \\
& \text { slowly. }
\end{aligned}
$$

This strategy was more obvious for "finding the line" task.

T6-P2: We went much slower when we were trying to find the line, but when this (feel the line) was, it was really like a nice clear path and the robot wasn't trying to like, stop you.

Below we describe some of the commonly observed collaboration strategies among the participants.

Corrective haptic feedback induces physical disagreement that encourages leader-follower roles exchange: Corrective haptic feedback requires both start time and speed coordination. For the case of corrective haptic feedback this means that the leader- defined as a person who initiates the movement or moves at a higher speed- always experiences forces against their intended trajectory of the robot movement.

\section{T5-P1: It is fighting me! Go! Go! Go! \\ T6-P1: ...it was like stopping every time we did a thing...}

On the other hand, the follower experiences encouraging forces to start moving or speeding up. This is reflected in the verbal discussion as a "go-stop-go" behaviour, in which the leader moved, the team did not see the intended results and so stopped the movement. This led to a verbal discussion around the strategy and opened up an opportunity for the follower to express his/her strategy and take the lead, which began a co-ordination, with the follower moving and so on.

T4-P2: Wait, can I try something? Okay. Is this the line that goes in this way? It keeps on pulling me back. It is moving by itself.

T4-P1: Can I try? T4-P2: You try. T4-P1: Put them back in there

Learners experienced many loops of go-stop-go while using the corrective haptic feedback which can explain the 
observed high role switching among participants, especially in the find the line task, during which they were still getting used to the co-ordination. We observe some frustration, especially for the find the line task in which performing the task solely relies on understanding the haptic feedback. However the co-ordination became smoother during the assistive feedback of the "feel the line" task, but was better demonstrated during the corrective feedback of the "draw the line" task.

T6-P1 Oh, I'm sorry. Oh, it really doesn't want, it doesn't want to do this kind of going.

T6-P2:It's going and stopping.

T6-P1: It's not doing what it was doing before (find the line) where it was being annoying.

Followers can be asked to lead the physical movements: One strategy that taken by one participant was to ask his partner to take the lead on moving the robot and then coordinating himself with her movements.

T3-P2: Okay, now move it one time. Three, two, one go. Oh, I have to start here. Hold up. Go again. Wait, I have to start here. Go again... wait. Three, two, one, go. We got this. We are drawing an X.

\section{E. Discussion}

To summarize the research outcomes, our qualitative and quantitative findings together show that the haptic feedback supported collaboration and co-ordination among learners. Depending on the activity (find vs feel vs draw), learners developed different collaboration strategies to leverage the haptic feedback appropriately. To reflect on the first research question of Study 2, our quantitative analysis of robot movement together with the qualitative analysis of learners conversation suggest that participants use both the haptic feedback and the verbal channels to collaborate and coordinate during the activity. While the primary use of verbal channel was to initiate the collaboration, the haptic feedback shaped the collaboration strategies such as comovement and turn-taking (qualitative and quantitative findings) to complete the activity. To answer the second research question, our quantitative findings suggest that participants adapted their individual strategies depending on the activity as seen by the variation in the haptic agreement with activity type. Furthermore, we found that the learners collaboration and co-ordination strategies also varied depending on the task. In the "find" task, learners were still discovering the role of the haptic and so there was greater disagreement (qualitative findings), low co-movement, high accuracy error and velocity (quantitative findings). In the "feel" task with assistive force feedback the agreement (qualitative findings) and co-movement were high (quantitative findings), while the accuracy error and velocity decrease. Finally, in the "draw" task, learners developed collaboration strategies (moving slowly and taking turns) to use the corrective feedback to do the activity better as seen from their conversations, high co-movement, low turn-taking, low accuracy error and low velocity.

\section{CONCLUSION}

In this work we investigate the role that haptics, and specifically, haptic feedback provided by handheld robots, can play in the context of collaborative learning.

To frame our research, we designed a collaborative learning activity with the goal to give students an intuitive understanding on the notion of functions and a way to represent them graphically, which demonstrated a positive learning gain even with a small duration of 40 minutes. Our results showed that while haptic feedback might not have a direct effect on learning, it can indirectly support it, by encouraging the interaction with the robot which, in turn, seems to be beneficial for learning. During a second study, we further investigated the role of haptics in the collaboration between learners. Quantitative and qualitative analyses were conducted and showed that the haptic channel is indeed used for different collaboration strategies such as coordination in movements, or the exchange of leader-follower roles. Specifically, haptic feedback changed the task, and the goal became to develop a strategy that allowed them to benefit from the haptic feedback to coordinate their moves and collaborate on the learning activity.

Besides the promising findings, we are aware of some limitations of this work. The context of this activity is one specific mathematical context; going beyond a unique context could be a potential addition and future work. Moreover, since the activity lasted only for one session, the novelty effect and learning curve related to the activity, the use of Cellulo robots with haptic feedback could all have played a role as confounding factors. Lastly, the low number of participants in both studies calls for extensions with larger sample sizes.

Building on our findings, we believe that further work should be conducted to investigate the inter-relationships between multi-modal communication channels (audio, visual and haptic), interaction patterns and learning outcomes. Similarly, the findings in Study 2 suggest that haptic feedback, rather than having a unique role in collaborative learning activities, might actually play different roles according to the nature of the task in which it is used. Along this line, future work should further investigate the links connecting task features to the learners' use of the haptic feedback and learning outcomes. Our short post-experiment survey on the motivation and usability of the device suggests perceivable force, high enjoyment, and interactivity of the robots. All participants except one who found the robot confusing score 5-7 (7 point Likert scale) and found the robots useful for learning math concepts.

\section{ACKNOWLEDGEMENTS}

We would like to thank all the students who participated in our experiment, the summer camp organizers, the school and the teachers who helped us organize the logistics of the study. The authors want to thank Swiss National Science Foundation through the National Centre of Competence in Research Robotics for their supports and Mitacs Globalink 
Research Award for helping graduate student doing the relevant research abroad.

\section{REFERENCES}

[1] P. Dillenbourg, "What do you mean by collaborative learning?" Collaborative-learning: Cognitive and Computational Approaches, vol. Vol. 1, 011999.

[2] J.-W. Strijbos, "Assessment of (Computer-Supported) Collaborative Learning," IEEE Transactions on Learning Technologies, vol. 4, no. 1, pp. 59-73, Jan. 2011.

[3] M. Shimada, T. Kanda, and S. Koizumi, "How can a social robot facilitate children's collaboration?" in Social Robotics, S. S. Ge, O. Khatib, J.-J. Cabibihan, R. Simmons, and M.-A. Williams, Eds. Berlin, Heidelberg: Springer Berlin Heidelberg, 2012, pp. 98-107.

[4] L. El Hamamsy, W. Johal, T. Asselborn, J. Nasir, and P. Dillenbourg, "Learning by collaborative teaching: An engaging multi-party cowriter activity," in 2019 28th IEEE International Conference on Robot and Human Interactive Communication (RO-MAN). IEEE, 2019, pp. 1-8.

[5] R. B. Rosenberg-Kima, Y. Koren, and G. Gordon, "Robot-Supported Collaborative Learning (RSCL): Social Robots as Teaching Assistants for Higher Education Small Group Facilitation," Frontiers in Robotics and AI, vol. 6, 2020.

[6] J. Wainer, E. Ferrari, K. Dautenhahn, and B. Robins, "The effectiveness of using a robotics class to foster collaboration among groups of children with autism in an exploratory study," Personal and Ubiquitous Computing, vol. 14, p. 445-455, 2010.

[7] A. Özgür, S. Lemaignan, W. Johal, M. Beltran, M. Briod, L. Pereyre, F. Mondada, and P. Dillenbourg, "Cellulo: Versatile handheld robots for education," in Proceedings of the 2017 ACM/IEEE International Conference on Human-Robot Interaction. ACM, 2017, pp. 119-127.

[8] D. D. Suthers, "Technology affordances for intersubjective meaning making: A research agenda for cscl," International Journal of Computer-Supported Collaborative Learning, vol. 1, no. 3, pp. 315337, 2006.

[9] J. Chen, M. Wang, P. A. Kirschner, and C.-C. Tsai, "The role of collaboration, computer use, learning environments, and supporting strategies in cscl: A meta-analysis," Review of Educational Research, vol. 88, no. 6, pp. 799-843, 2018.

[10] E. Lehtinen, K. Hakkarainen, L. Lipponen, M. Rahikainen, and H. Muukkonen, "Computer supported collaborative learning: A review," The JHGI Giesbers reports on education, vol. 10, 1999.

[11] J. Wang, A. Chellali, and C. G. L. Cao, "Haptic communication in collaborative virtual environments," Human Factors, vol. 58, no. 3, pp. 496-508, 2016, pMID: 26715689.

[12] G. Sankaranarayanan and B. Hannaford, "Experimental internet haptic collaboration using virtual coupling schemes," in 2008 Symposium on Haptic Interfaces for Virtual Environment and Teleoperator Systems, 2008, pp. 259-266.

[13] J. Kim, H. Kim, B. K. Tay, M. Muniyandi, M. A. Srinivasan, J. Jordan, J. Mortensen, M. Oliveira, and M. Slater, "Transatlantic touch: A study of haptic collaboration over long distance," Presence: Teleoper. Virtual Environ., vol. 13, no. 3, p. 328-337, Jul. 2004.

[14] J. Smith and K. MacLean, "Communicating emotion through a haptic link: Design space and methodology," International Journal of Human-Computer Studies, vol. 65, no. 4, pp. 376-387, Apr. 2007.

[15] I. Oakley, S. Brewster, and P. Gray, "Can You Feel the Force? An Investigation of Haptic Collaboration in Shared Editors," in In Proceedings of Eurohaptics, 2001, pp. 54-59.

[16] E.-L. Sallnäs, K. Rassmus-Gröhn, and C. Sjöström, "Supporting presence in collaborative environments by haptic force feedback," ACM Trans. Comput.-Hum. Interact., vol. 7, no. 4, p. 461-476, Dec. 2000.

[17] S. Kianzad and K. E. MacLean, "Collaborating through magic pens: Grounded forces in large, overlappable workspaces," in Haptic Interaction. Singapore: Springer Singapore, 2019, pp. 233-237.

[18] T. Asselborn, A. Guneysu, K. Mrini, E. Yadollahi, A. Ozgur, W. Johal, and P. Dillenbourg, "Bringing letters to life: Handwriting with hapticenabled tangible robots," in Proceedings of the 17th ACM Conference on Interaction Design and Children, 2018, p. 219-230.

[19] W. Johal, S. Andersen, M. Chevalier, A. Ozgur, F. Mondada, and P. Dillenbourg, "Learning symmetry with tangible robots," in Robotics in Education. Springer International Publishing, 2019, pp. 270-283.

[20] A. Guneysu Ozgur, A. Özgür, T. Asselborn, W. Johal, E. Yadollahi, B. Bruno, M. Skweres, and P. Dillenbourg, "Iterative design and evaluation of a tangible robot-assisted handwriting activity for special education," Frontiers in Robotics and AI, vol. 7, p. 29, 2020.
[21] A. Magana and S. Balachandran, "Unpacking students' conceptualizations through haptic feedback," Journal of Computer Assisted Learning, vol. 33, no. 5, pp. 513-531, 2017.

[22] G. Hallman, I. Paley, I. Han, and J. B. Black, "Possibilities of haptic feedback simulation for physics learning," in Proceedings of EdMedia + Innovate Learning 2009, June 2009, pp. 3597-3602.

[23] "Incorporating haptic feedback in simulation for learning physics," Computers Education, vol. 57, no. 4, pp. 2281 - 2290, 2011.

[24] R. L. Williams, M.-Y. Chen, and J. M. Seaton, "Haptics-augmented simple-machine educational tools," Journal of Science Education and Technology, vol. 12, no. 1, pp. 1-12, 2003.

[25] E. N. Wiebe, J. Minogue, M. G. Jones], J. Cowley, and D. Krebs, "Haptic feedback and students' learning about levers: Unraveling the effect of simulated touch," Computers Education, vol. 53, no. 3, pp. $667-676,2009$.

[26] M. Eid, M. Mansour, A. El Saddik, and R. Iglesias, "A haptic multimedia handwriting learning system," 01 2007, pp. 103-108.

[27] G. Minaker, O. Schneider, R. Davis, and K. E. MacLean, "Handson: enabling embodied, creative stem e-learning with programming-free force feedback," in International Conference on Human Haptic Sensing and Touch Enabled Computer Applications, 2016, pp. 427-437.

[28] G. Baud-Bovy and N. Balzarotti, "Using force-feedback devices in educational settings: A short review," in Proceedings of the 1st ACM SIGCHI International Workshop on Multimodal Interaction for Education, 2017, p. 14-21.

[29] M. Darrah, W. A. Aviles, K. Murphy, and K. Speransky, "Computer haptic effects in math and science lessons support student learning," 2015.

[30] R. L. Davis, M. Orta Martinez, O. Schneider, K. E. MacLean, A. M. Okamura, and P. Blikstein, "The haptic bridge: Towards a theory for haptic-supported learning," in Proceedings of the 2017 Conference on Interaction Design and Children. New York, NY, USA: Association for Computing Machinery, 2017, p. 51-60.

[31] J. Minogue, G. Jones, B. Broadwell, and T. Oppewall, "The impact of haptic augmentation on middle school students' conceptions of the animal cell," Virtual Reality, vol. 10, pp. 293-305, 112006.

[32] G. Jones, T. Andre, R. Superfine, and R. Taylor, "Learning at the nanoscale: The impact of students' use of remote microscopy on concepts of viruses, scale, and microscopy," Journal of Research in Science Teaching, vol. 40, pp. 303 - 322, 032003.

[33] Z. C. Zacharia, "Examining whether touch sensory feedback is necessary for science learning through experimentation: A literature review of two different lines of research across k-16," Educational Research Review, vol. 16, pp. 116 - 137, 2015.

[34] S. Price and S. Duffy, "Opportunities and Challenges of Bodily Interaction for Geometry Learning to Inform Technology Design," Multimodal Technologies and Interaction, vol. 2, no. 3, p. 41, Sep. 2018.

[35] N. Yiannoutsou, R. Johnson, and S. Price, "Exploring How Children Interact with 3D Shapes Using Haptic Technologies," in Proceedings of the 17th ACM Conference on Interaction Design and Children, ser. IDC '18. New York, NY, USA: ACM, 2018, pp. 533-538, eventplace: Trondheim, Norway.

[36] S. Ghisio, E. Volta, P. Alborno, M. Gori, and G. Volpe, "An Open Platform for Full-body Multisensory Serious-games to Teach Geometry in Primary School," in Proceedings of the 1st ACM SIGCHI International Workshop on Multimodal Interaction for Education, 2017, pp. 49-52.

[37] S. Gerofsky, "Mathematical learning and gesture: Character viewpoint and observer viewpoint in students' gestured graphs of functions," Gesture, vol. 10, pp. 321-343, 012010.

[38] A. Özgür, W. Johal, F. Mondada, and P. Dillenbourg, "Haptic-enabled handheld mobile robots: Design and analysis," in Proceedings of the 2017 CHI Conference on Human Factors in Computing Systems. ACM, 2017, pp. 2449-2461.

[39] P. Dillenbourg, S. Järvelä, and F. Fischer, "The evolution of research on computer-supported collaborative learning," in Technology-enhanced learning. Springer, 2009, pp. 3-19.

[40] P. Dillenbourg and D. Traum, "Sharing solutions: Persistence and grounding in multimodal collaborative problem solving," The Journal of the Learning Sciences, vol. 15, no. 1, pp. 121-151, 2006.

[41] V. Clarke and V. Braun, "Thematic analysis," The Journal of Positive Psychology, vol. 12, no. 3, pp. 297-298, 2017. 